\title{
Impact of local anaesthetics and needle calibres used for painless PRP injections on platelet functionality
}

\author{
Olivier Bausset ${ }^{1}$ \\ Jeremy Magalon 1 \\ Laurent Giraudo1 \\ Marie-Laure Louis ${ }^{2}$ \\ Nicolas Serratrice ${ }^{3}$ \\ Corrine Frere ${ }^{4}$ \\ Guy Magalon ${ }^{3}$ \\ Françoise Dignat-George ${ }^{1,4}$ \\ Florence Sabatier ${ }^{1,4}$
}

1 Culture and Therapy Unit, Assistance Publique des Hôpitaux de Marseille, France

2 Orthopaedic Surgery Unit, CHU La Conception, Assistance Publique des Hôpitaux de Marseille, France

3 Department of Plastic and Reconstructive Surgery, CHU La Conception, Assistance Publique des Hôpitaux de Marseille, France

4 Faculty of Pharmacy, Inserm UMR-S1076, Marseille, France

Corresponding author:

Olivier Bausset

Culture and Therapy Unit, Assistance Publique des

Hôpitaux de Marseille

148 bd Baille

13005 Marseille, France

E-mail: olivier.bausset@wanadoo.fr

\section{Summary}

The platelet-rich plasma (PRP) is an autologous biotherapy commonly used for its healing properties. Once activated, platelets released a real "cocktail" of growth factor and cytokines implied in numerous regenerative processes. However the impact of medical practices associated to PRP therapeutic use on platelets functionality remains poorly known.

Objectives: we evaluated the in vitro effects of two commonly used local anesthetics (Xylocaine* and Naropin*) on PRP functionality. We also investigated the quantity and quality of PRP that passed through the smallest gauge needle commercialized.

Materials and methods: PRP from 9 healthy volunteers were prepared using our previously described home made purification protocol. Platelet aggregation capacity was evaluated by aggregometry assays and the growth factor release was determined by ELISA after platelet activation. We also evaluated the platelet activation status, reactivity and stability of platelets by flow cytometry using the P-selectin expression marker. Results: the association of local anaesthetics with PRP injections resulted in a significant decrease of platelets functionality, assessed by their capacity of aggregating. Local anaesthetics did not interfere with the growth factor release. The different needle sizes and calibres tested for PRP injections did not influence the platelet functionality.

Conclusions: the use of local anaesthetics to prevent pain during PRP injections could compromise the therapeutic potential of PRP. These results suggest using carefully local anaesthetics or limiting their use as often is possible. To minimize injection pain, we recommend using $30 \mathrm{G}$ needles. These data will lead to clinical recommendations for painless and controlled PRP injections.

KEY WORDS: platelet-rich plasma, PRP, Platelet concentrate local anaesthetics, growth factors, tendinopathy, sport medicine, regenerative medicine.

\section{Introduction}

The plasma enriched with platelets or platelet-rich plasma (PRP), is an autologous concentrated source of platelet. Once activated, degranulation happened and platelets released a real "cocktail" of growth factor and cytokines implied in numerous regenerative processes $^{1}$. These properties have been investigated and used as clinical tools for several types of medical treatments, including nerve injury, tendinitis, osteoarthritis, cardiac muscle injury, bone repair and regeneration, plastic surgery, and oral surgery. PRP has also received attention in the popular media for its applications in regenerative sport medicine $e^{2,3}$. PRP is basically purified by gentle centrifugation from whole peripheral blood. Several kits are available on the market providing different systems to purify the PRP. Even if it seems easy in appearance, numerous purification factors as the centrifugation speed, the use of anticoagulant can influence the properties of the final PRP product. The main modified properties are the presence of leucocytes or red blood cells, and the growth factor content. All these aspects are strongly discussed in the literature and currently there is no consensus. Moreover, many authors do not clearly document the characteristics of the product 
they are using, creating many misunderstandings. That's why there is no one PRP formulation, but a huge range of PRP formulations with different applications ${ }^{4}$. To add to the complexity, PRP content varies from one individual to another and within the same individual over the time leading to contrasting in vivo effects. Previously ${ }^{1}$, we developed a new PRP purification method based on two 15 minutes ( $\mathrm{min}$ ) successive centrifugations (respectively at 130 and $250 \mathrm{~g}$ ). In these conditions, we obtained a highly concentrated PRP product with very limited quantities of leukocytes (or white blood cells $=$ WBC) and red blood cells (RBC) suitable to study platelet properties.

In contrast, very little data exist on the effect of injections themselves on PRP properties. Lippi et al. ${ }^{5}$ described that the use of small-bore needles, could influence the platelet counting. That's the reason why we hypothesized that the needle size and calibre (expressed in Gauge $G$ ) could have a great influence on the quality of injected platelets.

In the same time, PRP injections have been noted to be painful, prompting clinicians to use local anaesthesia: Lidocaine with or without Epinephrine $(10 \mathrm{mg} / \mathrm{ml}$, $1 / 200.000)$ and Ropivacaine $(7.5 \mathrm{mg} / \mathrm{ml})$ are local anesthetics commonly used to prevent pain associated with PRP injection or for infiltration during total knee arthroplasty6-8. Schnabl et al. ${ }^{9}$ demonstrated that the longest pain reduction was shown for Ropivacaine $0.75 \%$ and Lidocaine with Epinephrine. Epinephrine is also used for its vasoactive capacity reducing bleeding. Moreover, the intra-articular or the intra-parietal use of Ropivacaine provided an excellent control of pain after surgery. Local infiltration of Ropivacaine demonstrated a significant reduction of hospitalization time, with an early return of motion (ROM), and a reduction of the main pain scores due to a significant decrease of the post-operative pain 7 . Local infiltration of Ropivacaine is simple, easy, safe, and effective for pain management after knee or hip surgery. However, data concerning on the effects of the addition of these local anaesthetics on platelet structure and functionality are lacking. Moreover, immediately after corticoids or PRP injections, the surrounding body fluids quickly diluted these substances ${ }^{10}$. This parameter is important and should be kept in mind in studies to get as close as possible to the in vivo conditions.

Here we aimed to analyze some information about the in vitro effects of two local anesthetics commonly used on PRP properties. We also investigated the quantity and quality of platelets from our home made PRP that passed through the smallest gauge needle commercialized. Hence we determined the best size needle for painless injections without platelet alteration.

To explore these two conditions (local anesthesia using and needle size) on platelets quality, we performed platelet aggregation assays and growth factor quantification. We also evaluated the platelet activation using the P-selection membrane marker by flow cytometry analysis. These pave the way for clinical recommendations for painless and controlled PRP injections.

\section{Materials and methods}

\section{Whole-blood collection}

Nine healthy volunteers donors ( 4 men and 5 women) aged between 22-63 year old (mean 36.2 years) entered the study. Volunteers were included in the study after informed consent was obtained according to national rules. They had no relevant diseases and had not taken any drugs known to affect platelet function for 7 days prior to the study. For each donor, $34 \mathrm{ml}$ of whole blood were collected in four 10 ml-tubes (Vacuette ${ }^{\circledR}$, Ref 455055 , Greiner Bio-One SAS) containing $1.5 \mathrm{ml}$ of Adenosin citrate dextrose-acid (ACD-A) solution (Fenwal, ref BDB8651), for a final volume of $40 \mathrm{ml}$. Another tube, coated with Ethylenediaminetetraacetic acid (EDTA) anticoagulant was used to determine the initial platelet number from the whole blood with an automatic cell counter (Advia 2120®, Siemens Diagnostic Solutions, Tarrytown, NY). All donors included in this study had initial platelet count over 150,000 platelets $/ \mu$ l.

\section{PRP production}

PRP were produced using a previously developed new PRP purification method based on two $15 \mathrm{~min}$ successive centrifugations (respectively at $130 \mathrm{~g}$ and $250 \mathrm{~g})^{1}$. In these conditions, we obtained approximately $4 \mathrm{ml}$ of a highly concentrated PRP product with very limited quantities of RBC and WBC. After PRP purification, a $250 \mu \mathrm{l}$ aliquot was collected for a platelet count (Advia 2120®, Siemens Diagnostic Solutions, Tarrytown, NY). We also determined simultaneously residual RBC and WBC numeration. Platelets concentration factor was calculated relative to peripheral blood. $\mathrm{pH}$ was measured. Sterility assays were performed. All manipulations for PRP preparation and sampling were conducted under class A microbiological safety facility located in the Cell Therapy Unit at La Conception Hospital (AP-HM, France). Under these conditions we obtained a controlled cellular therapy product, optimized for human medical applications.

\section{Local anaesthetics}

Lidocaine and Ropivacaine are local anaesthetics belonging to the group of $\mathrm{N}$-alkylproline anilides. Xylocaine $(10 \mathrm{mg} / \mathrm{ml}$, AstraZeneca) with or without Epinephrine $(1: 200,000)$ : each $\mathrm{ml}$ of solution contained Lidocaine hydrochloride monohydrate equivalent to $10 \mathrm{mg}$ of Lidocaine hydrochloride anhydrous $(200 \mathrm{mg}$ per $20 \mathrm{ml}$ vial), 5 micrograms $(\mu \mathrm{g})$ of Epinephrine $(100 \mu \mathrm{g}$ per $20 \mathrm{ml}$ vial). Naropeine $(7.5 \mathrm{mg} / \mathrm{ml}$, AstraZeneca): each $\mathrm{ml}$ solution contained $7.5 \mathrm{mg}$ of Ropivacaine hydrochloride (150 mg per $20 \mathrm{ml}$ vial). We used a diluted concentration to replicate in vivo conditions ${ }^{6,10}$ corresponding to $20 \mu$ l of local anaesthetics in $240-\mu$ l cartridges. Final concentrations in 
the experiment are $0.83 \mathrm{mg} / \mathrm{ml}$ for Lidocaine and $0.625 \mathrm{mg} / \mathrm{ml}$ for Naropeine.

\section{Aggregometry}

The effects of local anaesthetics on platelet aggregation were determined by light transmittance aggregometry. Platelet aggregation was assessed with an APACT 4004 Aggregometer (Elitech, France). Single-use cartridges were used. A final volume of $240 \mu \mathrm{l}$ of PRP (adjusted for a platelet number of $250 \mathrm{G} / \mathrm{l}$ diluted in Platelet Poor Plasma (PPP) was used to test the different conditions (Tab. 1). Aggregation was initiated at $37^{\circ} \mathrm{C}$ under gentle agitation by the addition of $20 \mu$ of Adenosine diphosphate (ADP) (100 $\mu \mathrm{M}$, Helena Biosciences Europe) or collagen $(190 \mu \mathrm{g} / \mathrm{ml}$, Helena Biosciences Europe). $240 \mu$ l of PRP was used as negative control to determine the basal platelet auto-aggregation. In these conditions, local anaesthetics were diluted (ratio 1/12) to mimic physiological conditions ${ }^{6,10}$. Phosphate buffer saline (PBS) was used as negative control. Light transmission was recorded during $300 \mathrm{~s}$ and analysed using software program (APACT Software). PRP aggregation was expressed on maximal intensity of platelet aggregation. We performed one assay for each sample.

\section{Growth factor quantification}

Growth factor release was evaluated after platelet activation using a collagen plate. BD BioCoat ${ }^{\mathrm{TM}}$ Cell Environments Collagen I 48-well plates (Becton Dickinson Labware, Bedford, UK, ref: 354505) were used to compare the release of growth factor. For each donor, $200 \mu$ of PRP (platelet concentration: $250 \mathrm{G} / \mathrm{l}$ diluted in PPP) and $20 \mu \mathrm{l}$ of PBS or Lidocaïne without Epinephrine were incubated at $37^{\circ} \mathrm{C}$ during $72 \mathrm{~h}$. Then supernatants were collected by gentle aspiration and were stored at $-80^{\circ} \mathrm{C}$ until quantification of growth factor (PDGF-AB, TGF- $\beta 1$ ) using Enzymelinked immunosorbent assay (ELISA) kits (R\&D Diagnostics, Wiesbaden, Germany). Assays were performed according to the manufacturer's instructions.

\section{Flow cytometry}

The quantity and quality of platelets that passed through a $30 \mathrm{G}$ needle (the smallest gauge needle commercialized, length: $13 \mathrm{~mm}$ ) compared to non-injected PRP was evaluated by flow cytometry using the P-selectin marker. PRP was detected by CD62P monoclonal antibody labelled with FITC (clone LYP20, Biocytex, Marseilles, France). A FITC conjugated isotype antibody (clone 2DNP2H11, Biocytex) was used as control. After 20 min of incubation, platelets were resuspended in $500 \mu$ l of appropriate saline buffer and analysed with a FC-500 cytometer (Beckman Coulter, Miami, FL, USA). Light scatter and fluorescence channels set up at logarithmic gain and platelets gated according to forward and side scatter properties. P-selectin positive $(+)$ expression was detected over non-specific background signal from isotype control labelling. A total of 10,000 events were monitored. CXP software (Beckman Coulter) was used for data processing. Results were expressed as rate of platelet with P-selectin expression (the Mean Fluorescence Intensity of the specific labelling of the entire platelet populations).

\section{Statistical analysis}

Statistical analyses were performed with GraphPad Prism 6.0 software. A value of $p<0.05$ considered as significant. All quantitative measurements described using summary statistics ( $\mathrm{n}$, mean, standard deviation). The Mann-Whitney test was used to analyze the possible influence of local anaesthetics and needle calibres on the platelet functionality.

\section{Results}

\section{Effect of the local anaesthetics on platelet aggre- gation}

The effects of local anaesthetics on platelet functionality were evaluated by light transmittance aggregom-

Table 1. Light Transmittance aggregometry: the different experimental conditions.

\begin{tabular}{llll}
\hline$\#$ & PRP & Aggregation activators & Local anaesthetics \\
\hline $\mathbf{1}$ & $240 \mu \mathrm{l}$ & - & - \\
$\mathbf{2}$ & $200 \mu \mathrm{l}$ & $20 \mu \mathrm{l}$ of ADP & $20 \mu \mathrm{l}$ of PBS \\
$\mathbf{3}$ & $200 \mu \mathrm{l}$ & $20 \mu \mathrm{l}$ of ADP & $20 \mu \mathrm{l}$ of Lidocaine \\
$\mathbf{4}$ & $200 \mu \mathrm{l}$ & $20 \mu \mathrm{l}$ of ADP & $20 \mu \mathrm{l}$ of Lidocaine + Epinephrine \\
$\mathbf{5}$ & $200 \mu \mathrm{l}$ & $20 \mu \mathrm{l}$ of ADP & $20 \mu \mathrm{l}$ of Ropivacaine \\
$\mathbf{6}$ & $200 \mu \mathrm{l}$ & $20 \mu \mathrm{l}$ of collagen & $20 \mu \mathrm{l}$ of PBS \\
$\mathbf{7}$ & $200 \mu \mathrm{l}$ & $20 \mu \mathrm{l}$ of collagen & $20 \mu \mathrm{l}$ of Lidocaine \\
$\mathbf{8}$ & $200 \mu \mathrm{l}$ & $20 \mu \mathrm{l}$ of collagen & $20 \mu \mathrm{l}$ of Lidocaine + Epinephrine \\
$\mathbf{9}$ & $200 \mu \mathrm{l}$ & $20 \mu \mathrm{l}$ of collagen & $20 \mu \mathrm{l}$ of Ropivacaine \\
\hline
\end{tabular}

A final volume of $240 \mu \mathrm{l}$ of PRP containing $250 \mathrm{G} /$ platelets were used for the light transmittance aggregometry assays. ADP $(100 \mu \mathrm{M})$ or collagen $(190 \mu \mathrm{g} / \mathrm{ml})$ were used to initiate the platelet aggregation. PRP was used as negative control to determine the basal platelet auto-aggregation. Two local anesthetics commonly used for PRP injections: Lidocaine (10 $\mathrm{mg} / \mathrm{ml})$ with or without epinephrine $(5 \mu \mathrm{g} / \mathrm{ml})$ and Ropivacaine $(7.5 \mathrm{mg} / \mathrm{ml})$ were evaluated. PBS was used as negative control. Abbreviations: $\mathrm{PBS}=$ phosphate buffer saline, $\mathrm{ADP}=$ adenosine diphosphate. 
etry (Fig. 1). PBS did not interact on PRP aggregation in response to ADP or collagen agonists. A significant lower aggregation was observed with the Lidocaine with or without Epinephrine and Ropivacaine in response to $\operatorname{ADP}(p=0.0039)$ or collagen $(p=0.0313)$ agonists. Platelets aggregation assays indicated that Lidocaine with or without Epinephrine and Ropivacaine local anaesthetics had a negative effect on platelet aggregation rate induced by ADP and collagen. The use of Epinephrine combined to Lidocaine enhanced $(p<0.0005)$ platelet aggregation compared to a lonely-Lidocaine use.

\section{Growth Factor Quantification}

No statistically significant difference could be detected in the releasing of growth factor with Lidocaine compared to untreated PRP (PDGF-AB $p=0.79$ and TGF-Béta1 $\mathrm{p}=0.76$ ) (Fig. 2). Local anaesthetics

\section{Light transmittance aggregometry}

A

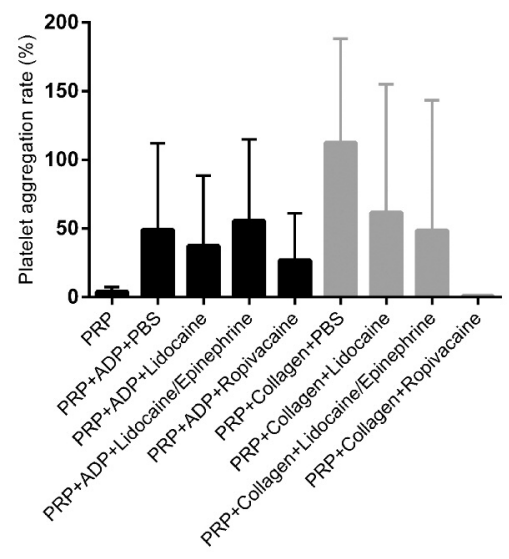

B

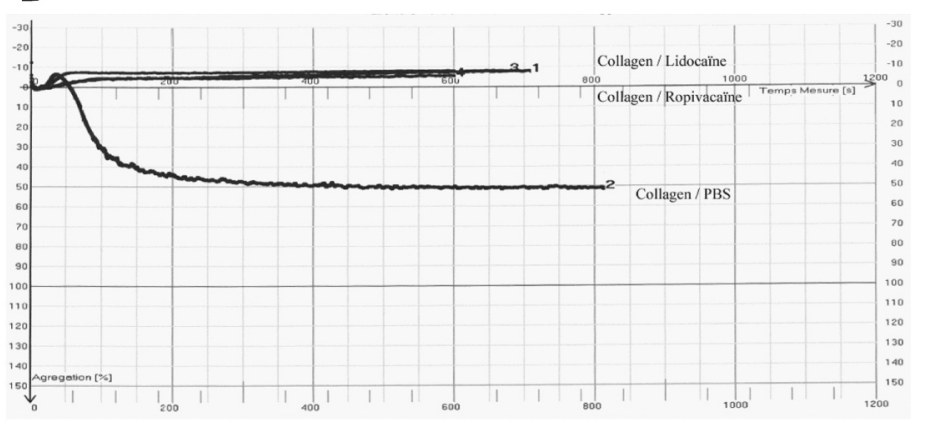

Figure 1. Light transmittance aggregometry with the standard deviation bar (A) Platelet aggregation rate (\%). (B) Platelet aggregation curve in response to collagen. Abbreviations: $\mathrm{PBS}=$ phosphate buffer saline, ADP $=$ adenosine diphosphate.

\section{Growth factor concentration}

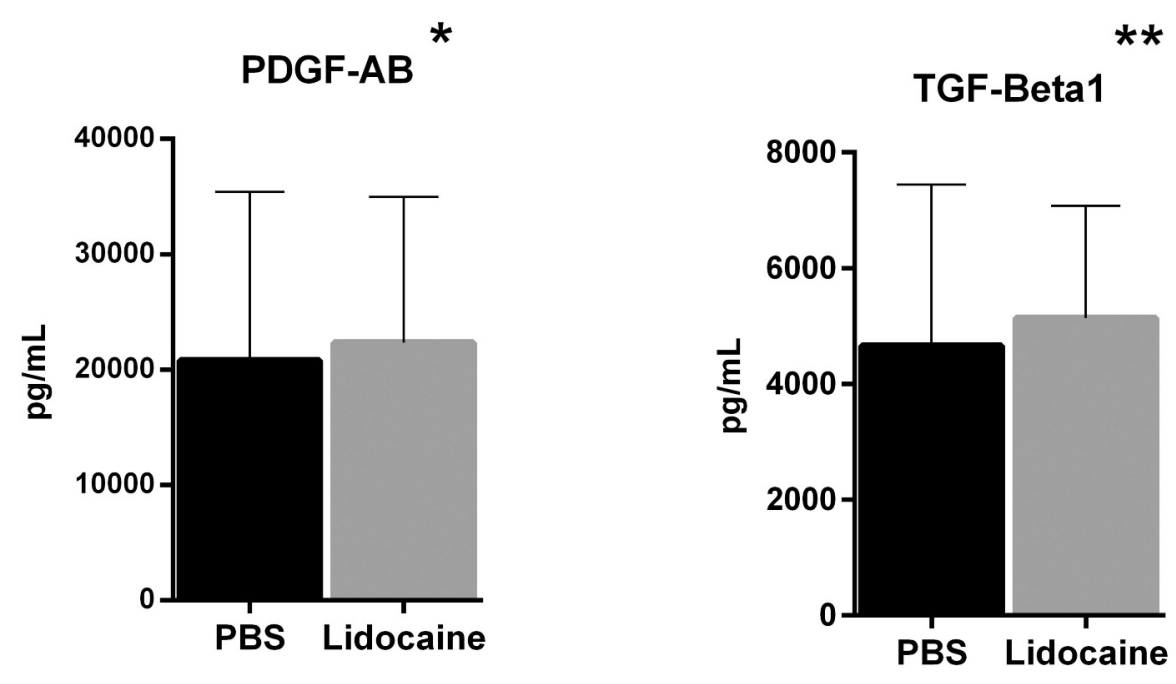

Figure 2. Growth factor concentrations (pg/ml). Data obtained for different seven donors. ${ }^{*} \mathrm{p}=0.79$ ** $\mathrm{p}=0.76$. PDGF-AB: platelet-derivated growth factor. TGF-Béta1: transforming growth factor $b 1 \mathrm{n}=7$. 
seemed to have no effect on the growth factor platelet release.

\section{Impact of the needle caliber on platelet activation status}

The platelet activation status was evaluated by flow cytometry using the P-selectin marker. We obtained $3.80+/-1.31 \%$ of activated platelets for the PRP that passed through a $30 \mathrm{G}$ needle and $3.37+/-1.18 \%$ for the untreated PRP. The smallest gauge needle commercialized did not appear to activate platelets, there was no statistically significant difference $(n=6, p=$ $0.41)$.

\section{Discussion}

In this study we showed that local anaesthetics could directly interfered with the platelet functionality, especially platelet aggregation. In contrary, local anaesthetics did not affect the capacity of PRP to release growth factor. Moreover, the smallest gauge needle csommercialized had no significant influence on the count and the quality of platelet from our home made PRP.

Previously Carofino et al. ${ }^{6}$ described that PRP injections combined with local anaesthetics were less effective than PRP injections without use of a local anaesthesia. Our results are consistent with the results of Az-ma et al. ${ }^{11}$ and Carofino et al. ${ }^{6}$. The use of local anaesthetics altered the platelet aggregation. Haalan et al. ${ }^{12}$ observed that Epinephrine enhanced platelet aggregation, that's why the Epinephrine seems to inhibit the negative effect of Lidocaine on the platelet aggregation. Adverse effects of anaesthetics on cells are not clearly understood. Local anaesthetics solutions are generally prepared at a $\mathrm{pH}$ of 5.0 to 6.0 , however this acidity may be detrimental to treated cells 6 . If the use of local anaesthetics is absolutely necessary, we encouraged to inject the local anaesthetics and the PRP in different sites to avoid any interactions. It is generally possible in many indications. As an example, for intra-articular injections the local anaesthesia is only limited to a local superficial skin area and the PRP is directly injected into the articular cavity. Platelets have never been directly in contact with the local anaesthetics. An alternative could be a short relaxation induced by MEOPA or a general anaesthesia.

No statistically significant difference has been observed for growth factor quantification. This result can be explained by a long time incubation. We suggest that a 72 hours-incubation in a collagen plate have been totally activating the platelets despite lidocaine incubation. During the first hours after collagen activation, it appears that lidocaine modifies the release kinetics of platelet growth factor.

The use of small-bore needles ( $30 \mathrm{G}$ ) had no influence on the quantity and the quality of platelets. This result is important because to reduce pain, clinicians used as it is possible small and atraumatic needles.
Indeed, 96\% of patients experienced a moderate post-procedural pain which lasted for $3 \pm 2$ days after PRP administration ${ }^{13,14}$. As reported by Rodrigues et al. ${ }^{13}$, considering intra-vitreal injections, the patients injected with the 26 or $27 \mathrm{G}$ needles experienced more pain matched to the 29 and $30 \mathrm{G}$.

There are several limitations of this study ${ }^{15}$.

The in vitro behaviour of platelets may not mimic the in vivo environment. It's difficult to reproduce environment injection conditions, especially for the local anaesthesia concentration. Other studies are necessary to confirm our results. An in vitro study on human tendon cells should be realised to examine the effects of mixing anaesthetics with PRP on cells proliferation and viability.

To analyze anaesthetics effects on release kinetics of platelet growth factor, we propose to quantify growth factor after platelet activation. This quantification could be performed on a collagen plate every 6 hours during 7 days.

Considering needle size, other length and calibre have to be studied. It's important to standardize the pressure application on the syringe piston. However according to Lancé et al. ${ }^{16}$, we suggest that the collection and injection methods do not seem relevant.

\section{Conclusion}

This study evidenced that use of local anaesthetics to prevent pain during PRP injections may compromise the potentially beneficial of PRP effects by reducing platelets reactivity. Accordingly, we suggest using carefully local anaesthetics or limiting their use as often as possible. In addition, since the different needle sizes and calibres tested for PRP injections were shown to have no influence on platelet functionality, we recommend using $30 \mathrm{G}$ needles to minimize injection pain. These data pave the way towards definition of recommended medical practices associated to PRP injections.

\section{Conflict of interest}

The authors state no conflict of interest.

\section{References}

1. Bausset $O$, Giraudo L, Veran J, et al. Formulation and storage of Platelet-Rich Plasma homemade product. Biores Open Access. 2012;1(3):115-123.

2. Lopez-Vidriero E, Goulding KA, Simon DA, Sanchez M, Johnson $\mathrm{DH}$. The use of platelet-rich plasma in arthroscopy and sports medicine: optimizing the healing environment. Arthroscopy. 2010;26(2):269-278.

3. Parafioriti A, Armiraglio E, Del Bianco S, Tibalt E, Oliva F, Berardi AC. Single injection of platelet-rich plasma in a rat Achilles tendon tear model. Muscles Ligaments Tendons J. 2011;1(2): 41-47.

4. Yuan T, Zhang C-Q, Wang JHC. Augmenting tendon and ligament repair with platelet-rich plasma (PRP). Muscles Ligaments Tendons J. 2013 3(3):139-149. 
5. Lippi G, Salvagno GL, Montagnana M, Poli G, Guidi GC. Influence of the needle bore size on platelet count and routine coagulation testing. Blood Coagul Fibrinolysis. 2006;17(7):557-561.

6. Carofino B CD, McCarthy MB, Bradley JP, et al. Corticosteroids and local anesthetics decrease positive effects of platelet-rich plasma: an in vitro study on human tendon cells. Arthroscopy. 2012;28(5):711-719.

7. Marret E, Gentili M, Bonnet MP, Bonnet F. Intra-articular ropivacaine $0.75 \%$ and bupivacaine $0.50 \%$ for analgesia after arthroscopic knee surgery: a randomized prospective study. Arthroscopy. 2005;21(3):313-316.

8. Samoladas EP, Chalidis B, Fotiadis H, Terzidis I, Ntobas T, Koimtzis M. The intra-articular use of ropivacaine for the control of post knee arthroscopy pain. J Orthop Surg Res. 2006:23;21:17.

9. Schnabl SM, Unglaub F, Leitz Z, Breuninger H, Häfner HM. Skin perfusion and pain evaluation with different local anaesthetics in a double blind randomized study following digital nerve block anaesthesia. Clin Hemorheol Microcirc. 2012. [Epub ahead of print].

10. Lippi G, Salvagno GL, Montagnana M, Poli G, Guidi GC. Influence of the needle bore size on platelet count and routine co- agulation testing. Blood Coagul Fibrinolysis. 2006;17(7):557561.

11. Az-ma $\mathrm{TH}$, Yuge $\mathrm{O}$. Inhibitory effect of Lidocaïne on cultured porcine aortic endothelial cell-dependent antiaggregation of platelets. Anesthesiology. 1995;83(2):374-381.

12. Haaland HD, Holmsen $\mathrm{H}$. Potentiation by adrenaline of agonist-induced responses in normal human platelets in vitro. Platelets. 2011;22(5):328-337.

13. Rodrigues EB, Grumann A Jr, Penha FM, et al. Effect of needle type and injection technique on pain level and vitreal reflux in intravitreal injection. J Ocul Pharmacol Ther. 2011;27(2): 197-203.

14. Ferrero G FE, Orlandi D, Martini C, et al. Ultrasound-guided injection of Platelet-Rich Plasma in chronic achilles and patellar tendinopathy. J Ultrasound. 2012(4):260-266.

15. Padulo J, Oliva F, Frizziero A, Maffulli N. Muscle, Ligaments and Tendons Journal. Basic principles and recommendations in clinical and field science research. MLTJ. 2013;4:250-252.

16. Lancé MD, Henskens YM, Nelemans P, et al. Do blood collection methods influence whole-blood platelet function analysis? Platelets. 2013;24(4):275-281. 